\title{
Some Effects on the Phases of Amalgam Induced by Corrosion
}

\author{
GENE A. HOLLAND* and KAMAL ASGAR \\ University of Michigan, Ann Arbor, Michigan 48104, USA
}

\begin{abstract}
"Aged" amalgam restorations were analyzed using X-ray spectrometry with the SEM and the electron microprobe. The results indicated that the corrosion process penetrates throughout the amalgam mass. The tin of the $\gamma_{2}$ phase consistently was associated with nonmetals, particularly chlorine, in areas of high corrosion. Other nonmetals were associated with tin within the interfacial corrosion layer and, to a lesser degree, within the amalgam bulk.
\end{abstract}

Alloys used in dentistry as restorative materials, like any metal, have a tendency to corrode electrochemically. The nature of dental amalgam to undergo a corrosion process that can lead to ultimate failure of the restoration particularly is important from a clinical standpoint. This failure usually manifests itself as either a bulk fracture of the restoration or as a progressive breakdown of the amalgam along the marginal angles. The resultant "marginal ditch" provides a nidus for the accumulation of microorganisms that renders the tooth-restoration interface subject to recurrent caries.

Factors other than corrosion, such as cavity design and manipulative variables, have been implicated in the deterioration of dental amalgam. However, the current status of amalgam research supports contentions that corrosion is the primary factor associated with the breakdown phenomenon.

The electrochemical behavior of dental amalgam and its constituent phases has received much attention in the past ten years. ${ }^{1-4}$ According to the theory advanced by Jorgensen ${ }^{5}$ and supported by Mateer and

This investigation was condensed from a thesis submitted in partial fufllment of the requirements for the MS degree in restorative dentistry at the University of Michigan, 1972.

Received for publication April 27, 1973.

- Present address: Department of Operative Dentistry, School of Dentistry, University of North Carolina, Chapel Hill, NC 27514.
Reitz, ${ }^{6}$ the corrosion process releases tin ions from the $\gamma_{2}\left(\mathrm{Sn}_{8} \mathrm{Hg}\right)$ phase, which then react with nonmetallic ions in saliva to produce tin salts.

Wei and Ingram ${ }^{7}$ used the electron microprobe to analyze the tooth-amalgam interface of several clinical specimens. The analyses showed high concentrations of tin in the corrosion products and migration of tin into dentin and enamel. Mateer and Reitz ${ }^{6}$ reported a layerlike corrosion product at the tooth-restoration interface, which suggested that the compounds formed gradually by reaction of the amalgam and oral fluids. Their $X$-ray diffraction analyses of bulk surface scrapings showed $\mathrm{Sn}_{2} \mathrm{~S}_{3}$ and $\beta \mathrm{SnO}_{2}$ as the corrosion products.

The purpose of this investigation was to evaluate some effects on the constituent phases of amalgam induced by corrosion. "Aged" amalgam restorations were analyzed qualitatively using the scanning electron microscope, the metallographic microscope, and the electron microprobe for elemental analyses.

\section{Materials and Methods}

SELECTION OF aMALGaM SPECIMENS.Twelve permanent posterior teeth containing aged amalgam restorations were selected for study. The term "aged" refers to restorations that had been in service for unknown lengths of time with varying degrees of deterioration. The restorations varied in degree of marginal breakdown, surface characteristics, and number of tooth surfaces covered.

SCANNING ELECTRON MICROSCOPE (SEM) EXAMINATION AND X-RAY ANALYSES OF CORROSION PRODUCTS ALONG THE TOOTH-AMALGAM INTERFACE.-Areas of the occlusal surface and paticularly the enamel-amalgam interfaces were examined with the SEM." A potential

a JSM-U3, JEOL, Japan Electron Optics Laboratory Co., Ltd., Bedford, Mass. 
of $20 \mathrm{kv}$ was used for all scans; the beam current was adjusted for each area analyzed to produce high resolution secondary electron images. An energy dispersive (discriminating) spectrometer system attached to the SEM was used to determine the elemental composition of bulk corrosion products of specimens at the tooth-amalgam interface. The potential of the SEM-detector system was maintained at $15 \mathrm{kv}$ for all analyses; the beam current was adjusted for each area analyzed to deliver a count rate of 800 counts per second. Two hundred second count times were used for all analyses.

METALlographic EXamination.-The specimens were prepared for optical microscopic examination in the following manner. Thin disks $^{\mathrm{b}}$ mounted on a modified jeweler's lathe were used to section the specimens vertically through the faciolingual dimensions of the crowns. The sectioning was performed at slow rotational speeds and under a stream of water to minimize heat generation within the specimens. Half of each sectioned specimen was embedded in an autopolymerizing casting resine and maintained at room temperature until the resin polymerized. The remaining section of each specimen was

b XA-1803-P-RR-5 rubber-bonded aluminum oxide wheel, 3-inch diameter with $1 / 2$-inch arbor hole, 0.013 inch thickness. Allison Co., Bridgeport, Conn.

e Crystal Clear Casting Resin, Fibre Glass-Evercoat Co., Inc., Cincinnati, Ohio.
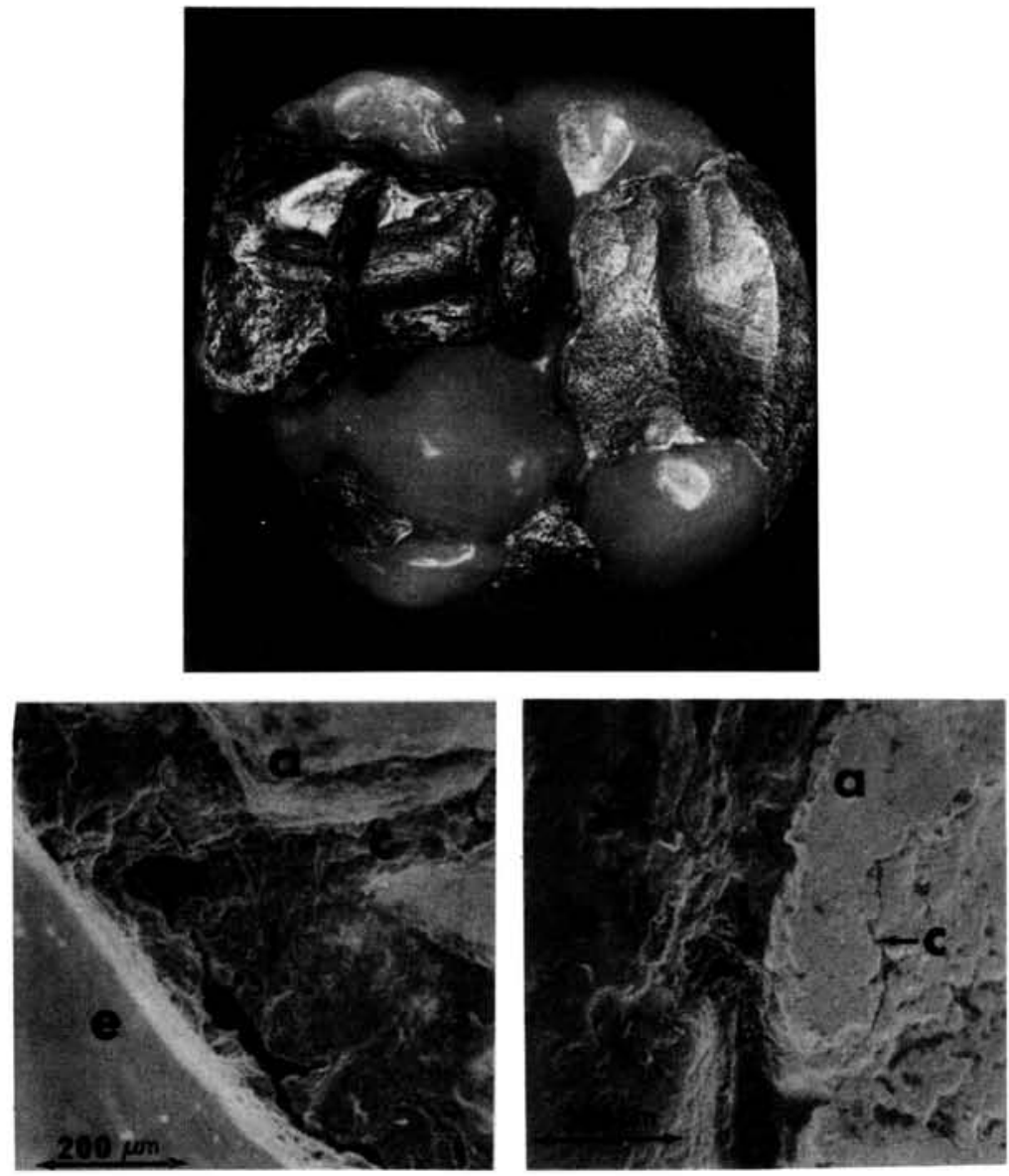

Fig 1.-Top, tooth specimen, occlusal view. Lower left, SEM image of interface (i) along lingual of MO restoration showing bulk transverse fracture (c) across central fossa (orig mag $\times 100$ ). Lower right, SEM image of interface along facial triangular ridge of DO restoration (orig mag $\times 100$ ). Note the pitting and microscopic crack (c). $e$, enamel; $a$, amalgam. 
saved for x-ray analysis of the interfacial corrosion products.

The mounted sections were polished metallographically under water using the normal procedures. ${ }^{8}$ The specimens were then examined at various magnifications under the metallographic microscope. ${ }^{d}$ Photomicrographs of representative structures were obtained.

ANALYSES OF CROSS-SECTIONS WITH ELECTRON MICROPROBE (EM).- Seven cross-sectioned specimens, previously polished metallographically for microscopic examination, were selected for chemical analysis with the electron microprobe. The chemical compositions of areas (phases), identified microscopically as atypical of freshly prepared amalgam, were determined by spot analyses. Adjacent areas of the surrounding matrix were analyzed for comparison. Single-channel analysis was used to produce X-ray distribution images for the principle elements of the atypical phases in some specimens. The potential of the system was held constant at $20 \mathrm{kv}$ for all specimens. Other instrument calibrations (count rate, count time, and beam current) were adjusted for each specimen. However, instrument variables were held constant for all areas analyzed within the same specimen.

\section{Results}

SEM Examination.-Scanning electron photomicrographs of representative areas along the enamel-amalgam interface of one specimen is shown in Fig 1. An occlusal photograph of the tooth is included at the top of the figure. The SEM images illustrate certain characteristics consistently ob. served in the 15 restorations examined.

The amalgam showed varying degrees of roughness, pitting and microscopic porosity at its surface, and generalized breakdown along the marginal angles as observed clinically. Surface pitting and porosity were concentrated in some areas at or near the enamel-amalgam interface of all restorations examined; however, both tended to be generally distributed across the surface of the amalgam. Microscopic cracks were observed along the marginal areas of some restorations (Fig 1, lower right). The crack appears to be penetrating through porosities.

Examination of the enamel-amalgam inter-

${ }^{d}$ Leitz Wetzler (620967), Ernst Leitz, W Ger. Calif. face showed a build up of a discontinuous mass, with a "mud-flake" appearance, in each of the specimens. This corrosion layer was observed along the entire occlusal interface of all restorations. The terms "corrosion layer" and "corrosion mass" will be used hereafter to describe the discontinuous mass at the tooth restoration interface. Although measurements were not made, considerable variations in thickness of the corrosion layer were observed between specimens and between areas within the same specimen.

MICROSCOPIC EXAMINATION OF POLISHED CROSS-SECTIONS.-Microscopic examinations of the unetched, polished cross-sections showed that the corrosion process had not been confined to the superficial structure of the restorations. There distinct structures, not typically observed in noncorroded amalgam, were observed consistently throughout the bulk in 14 of 15 restorations (Fig 2). In addition, corrosion products were evident as layers along the occlusal surface (Fig 3) and along the tooth-amalgam interface (Fig 4, left) of some specimens. The microstructure of one specimen seemed relatively typical of a freshly prepared, unetched amalgam (Fig 4 , right). However, the $\gamma_{2}$ phase in this specimen was more evident than that which can be observed in freshly prepared, unetched specimens.

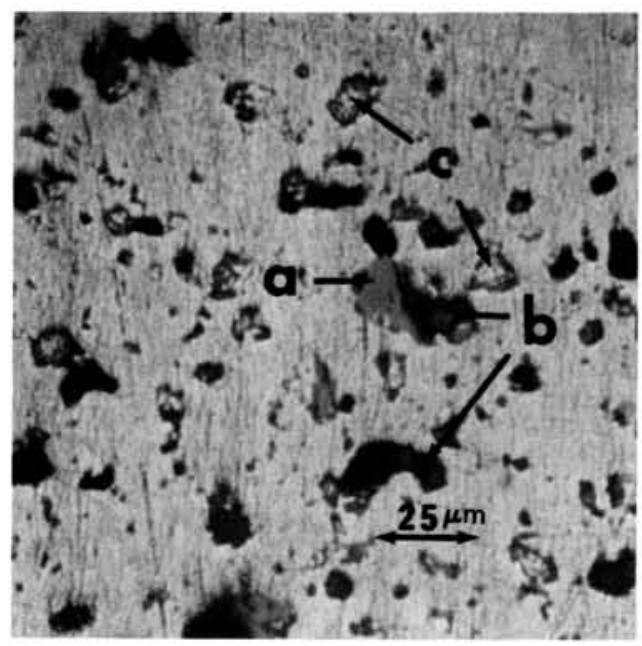

Fig 2.-Photomicrograph of polished cross-section (MO in Fig 1) demonstrates three phases associated with pentrating corrosion: $a$, bluegray phase; $b$, dark-gray phase; $c$, relatively noncorroded $\gamma_{2}$ phase (orig mag $\times 500$ ). 

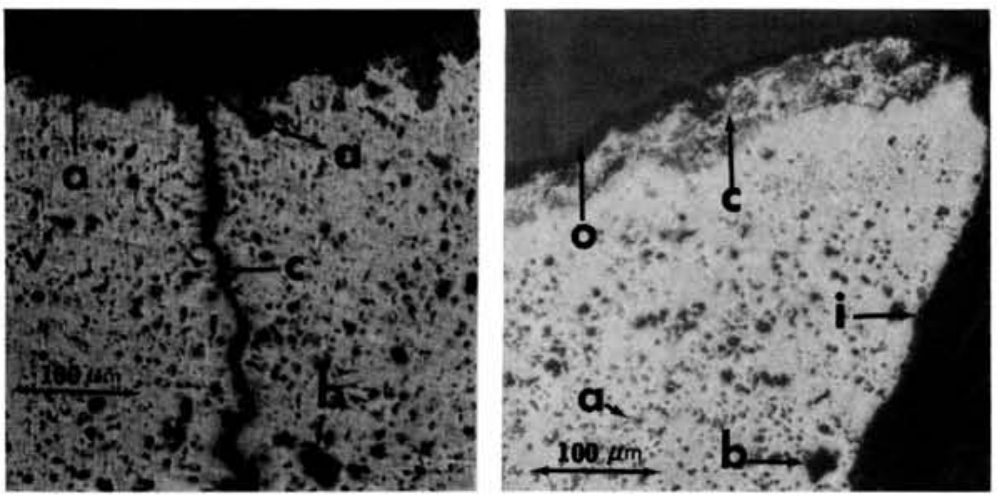

FIG 3.-Left, photomicrograph of polished cross-section (DO in Fig 1) demonstrates clustering of the blue-gray phase $(a)$ along occlusal $(o)$, vertical crack (c), and areas of dark-gray phase (b). v, void (orig mag $\times 200$ ). Right, photomicrograph of polished cross-section shows layered type corrosion $(c)$ along occlusal $(o)$, relatively noncorroded $\gamma_{2}(a)$, and the dark-gray phase $(b)$ near interface $(i)$. (orig mag $\times 200$ ).

Figure 5 shows a concentration of large and small dark-gray particles surrounded by a lighter matrix containing very small white crystals identified optically as $\gamma_{2}$. It is important to note that the $\gamma_{2}$ phase was diminished in areas with a high concentration of either the dark-gray phase or the bluegray phase. In these areas of high corrosion the $\gamma_{2}$ crystals seemed much smaller than those normally observed in freshly prepared amalgam. The dark gray areas appeared as voids partially filled with an amorphous-like material.

Another area within the same specimen
(Fig 6, left) shows a concentration of bluegray crystals near the tooth-amalgam interface. The structure and morphology of the blue-gray phase is more apparent in the $500 \times$ magnification of area $A$ in Fig 6 , left (Fig 6, right). The distribution of the bluegray phase followed a more consistent pattern than did that of the dark-gray phase.

Microscopic cracks within the amalgam structure tended to collect an amorphous material that had a similar appearance microscopically to that of the dark-gray phase (Fig 3).

X-RAY ANALYSIS OF INTERFACIAL CORROSION
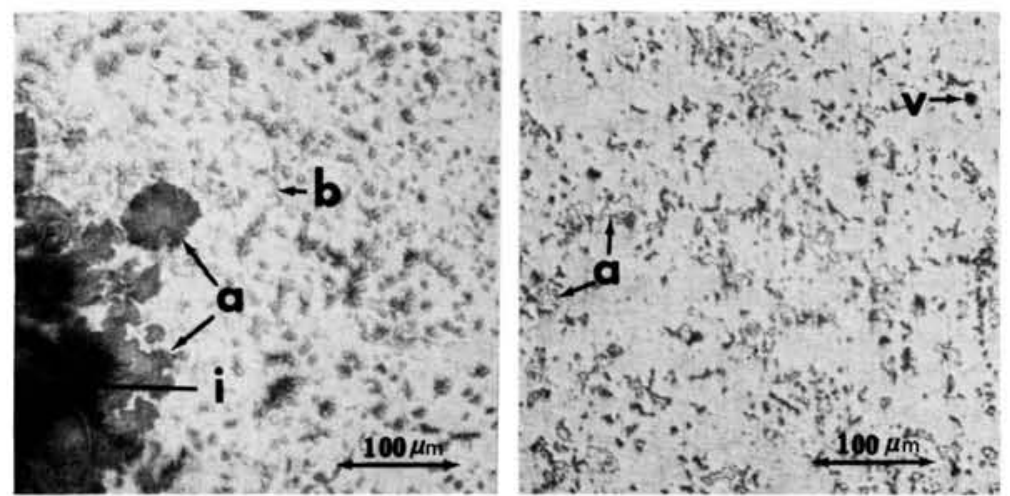

FIG 4.-Left, photomicrograph of polished cross-section (MO in Fig 1) shows clustered blue-gray phase (a) along interface (i), and small elongated crystals of noncorroded $\gamma_{2}(b)$. (orig mag $\left.\times 200\right)$. Right, photomicrograph of polished cross-section (DO in Fig 1) shows morphology and distribution of $\gamma_{2}$ (a) which was typical of areas examined in this specimen. $v$, void (orig mag $\times 200)$. 

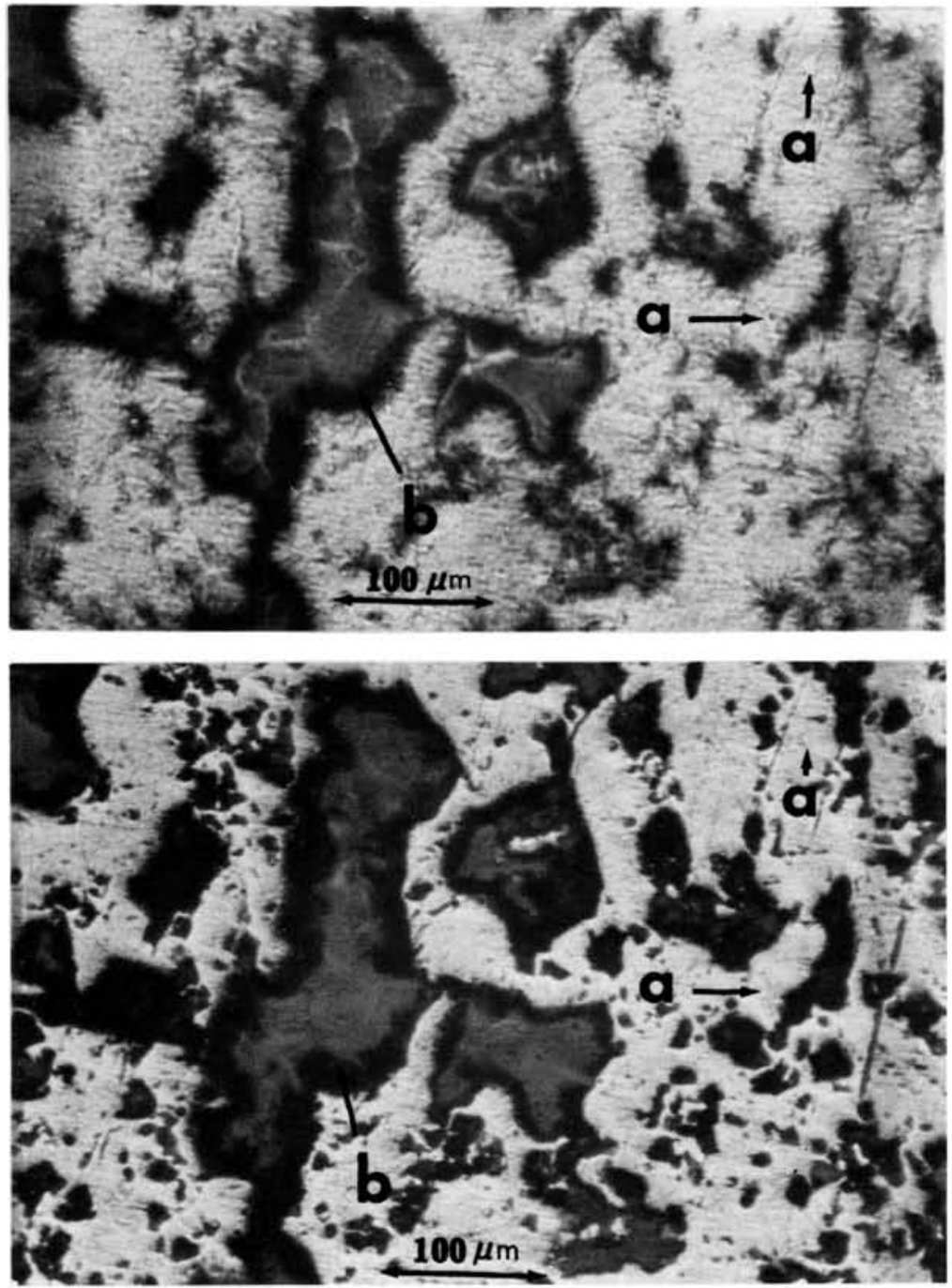

Fig 5.-Photomicrographs of polished and unetched cross-section show concentration of the dark-gray phase $(b)$ with surrounding very small crystals of $\gamma_{2}(a)$. Top, focus on $\gamma_{2}(a)$ and amorphous appearing material in the dark-gray phase $(b)$ (orig mag $\times 200$ ). Bottom, focus on matrix surrounding dark-gray phase.

PRODUCTs.-Figure 7 is a secondary electron image of a cross-sectioned restoration that shows the SEM appearance of interfacial corrosion products typical of the three specimens analyzed. The crazed character of the corrosion layer, as was observed in images of the enamel-amalgam interface on the occlusal surface, was also evident in images of the cavity surfaces of the restorations.

The results of the X-ray analyses of the corrosion layer varied somewhat, but a def- inite pattern of elemental distribution was evident. The bar graphs in Figure 8 illustrate the X-ray spectrum displays for representative areas of the corroded interface (top graph) and the cross-sectioned amalgam surface (bottom graph). Relative concentrations of the elements present are represented by the X-ray counts acquired. High counts for aluminum and silicon were consistently acquired in all analyses. These X-ray lines were attributed to a back reflection of X-rays 

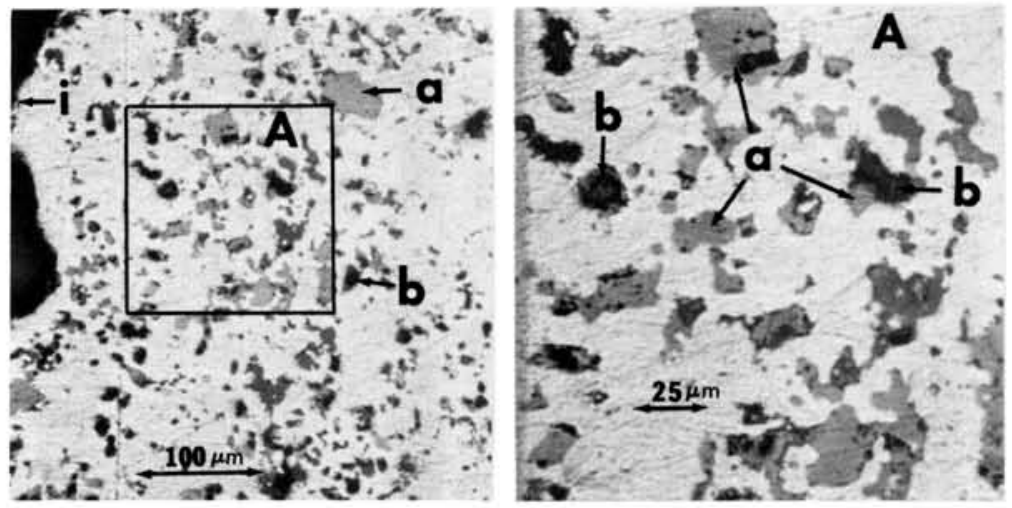

FIG 6.-Left, photomicrograph of polished cross-section shows high concentration of the blue-gray phase $(a)$ near interface $(i)$; b, dark-gray phase (orig mag $\times 200)$. Right, photomicrograph of area $A$ (orig mag $\times 500$ ) shows darkgray phase $(b)$ with surrounding blue-gray phase $(a)$.

from the mounting stubs that were of an aluminum alloy. ${ }^{9}$

Phosphorus (P), sulfur (S), and chlorine (Cl) were identified in most of the areas of the corrosion layers analyzed. Chlorine was present in three of eight areas of the cross-sectioned amalgam surfaces. The $\mathrm{Cl}$ concentration in these areas was higher than in comparable areas of the interfaces. The relative concentration of tin (Sn) was higher

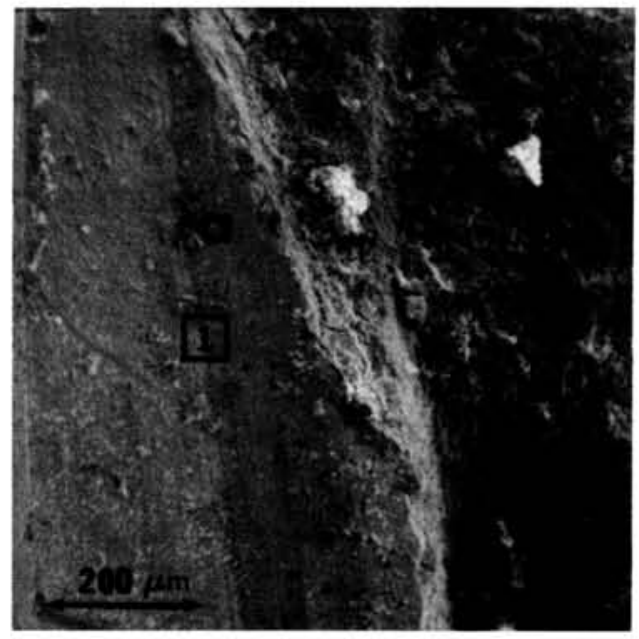

FIG 7.-SEM image of cross-sectioned amalgam surface (a) and interfacial corrosion products (i) of amalgam specimen which has had the surrounding tooth fractured away. Areas 1 and 2 are representative of areas analyzed with the solid-state X-ray detector system (orig mag $\times 100)$. in the corrosion layers than in the cross-sectioned surfaces. Conversely, the concentration of silver $(\mathrm{Ag})$ was higher within the amalgam than in the corrosion layers. Although mercury $(\mathrm{Hg})$ was identified in all areas of the cross-sectioned amalgams, its relative concentration varied among samples. Mercury could not be identified in the corrosion layer of any specimen. Copper $(\mathrm{Cu})$ and zinc $(\mathrm{Zn})$ were present in at least one area of each specimen within the corroded interface. Copper, but not $\mathrm{Zn}$, produced an
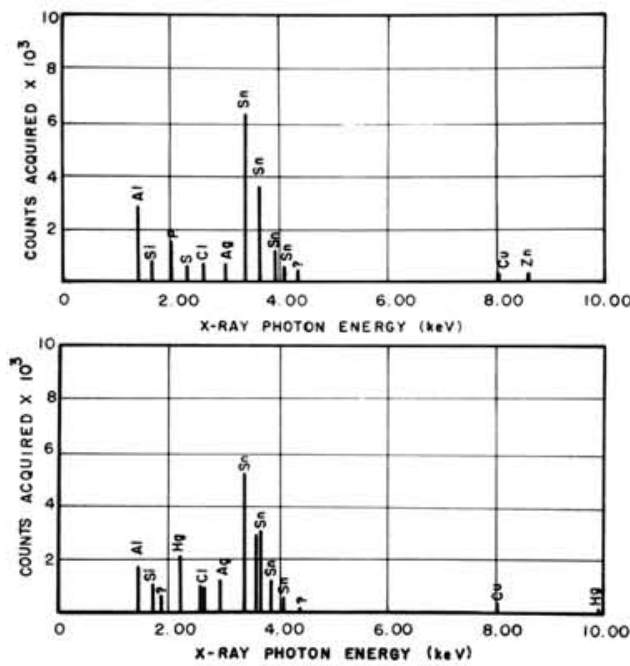

FIG 8.-Bar graphs illustrate the X-ray spectrum of elements identified in corroded interface $(t o p)$ and cross-sectioned amalgam surface (bottom). 

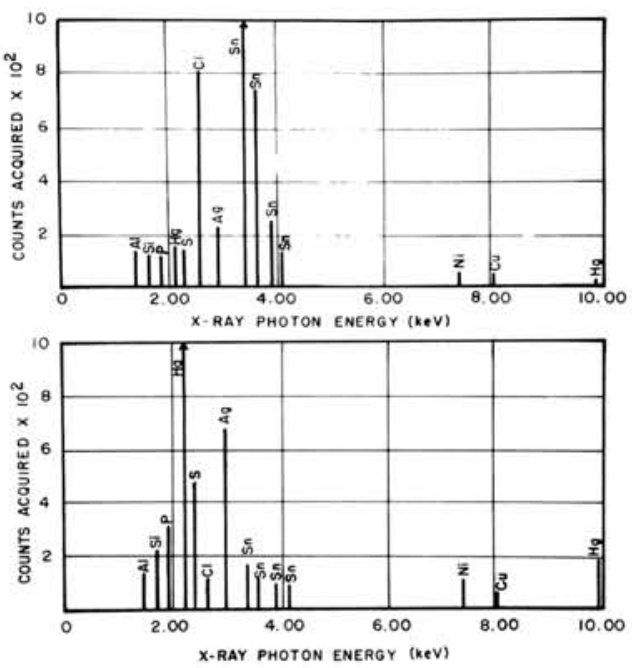

FIg 9.-Bar graphs illustrate the X-ray spectrum of elements identified in the dark-gray phase (top) and surrounding matrix (bottom). Arrows indicate that counts exceeded 1,000 counts full scale. identifiable X-ray line from the cross-sectioned amalgam.

ELECTRON MICROPROBE ANALYSES OF POLISHED CROSS-SECTIONS.-Because of the microscopic appearance including morphology, amount, and distribution of the dark-gray and blue-gray phases, the electron microprobe analyses were concentrated on these and adjacent areas of the matrix.

Typical X-ray data from analyses of the dark-gray phase and an adjacent matrix area are presented in Figure 9 in the form of bar graphs. Of particular interest was the presence of $\mathrm{P}, \mathrm{S}$, and $\mathrm{Cl}$ not only in the darkgray phase but also in the surrounding matrix in areas of some samples. However, the only consistency recorded regarding the presence of nonmetals was the high concentration of $\mathrm{Cl}$ in all of the dark-gray areas analyzed. A high concentration of Sn consis. tently was associated with the high $\mathrm{Cl}$ areas. A typical secondary electron image and $\mathrm{X}$ ray images for $\mathrm{Sn}$ and $\mathrm{Cl}$ from areas contain-

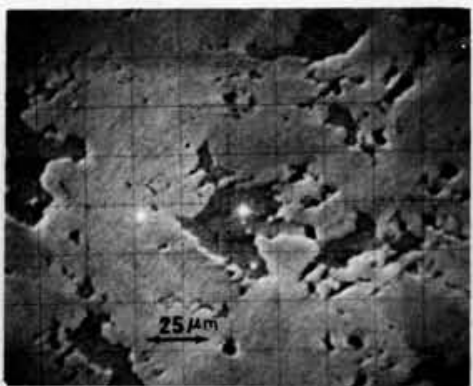

S.E.

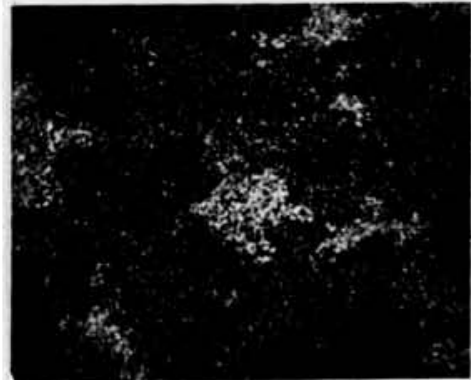

Sn

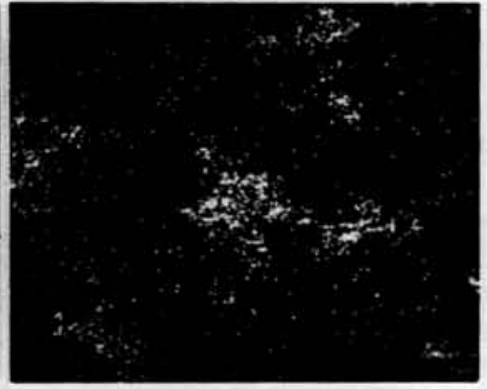

$\mathrm{Cl}$

Fig 10.-Images from electron microprobe analysis of a dark-gray phase (orig mag $\times 500$ ). 

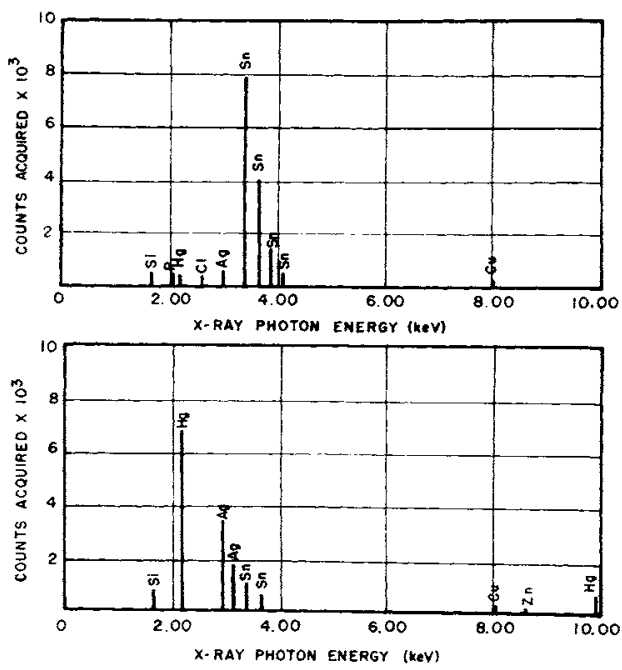

Fig 11.-Bar graphs illustrate X-ray spectrum of elements identified in the blue-gray phase (top) and surrounding matrix (bottom).

ing the dark-gray phase are presented in Figure 10. The X-ray images demonstrate the association of $\mathrm{Sn}$ and $\mathrm{Cl}$ throughout the dark-gray particles. Mercury and Ag concentrations were considerably lower in the dark-gray phase when compared to the surrounding matrix. No pattern was noted for the distribution of $\mathrm{Cu}$ and $\mathrm{Zn}$.

Figure 11 illustrates typical X-ray spectrum displays from the blue-gray phase (top graph) and the matrix surrounding the blue-gray phase (bottom graph) in the form of bar graphs. The blue-gray phase showed a high concentration of $\mathrm{Sn}$ relative to other elements present. On the other hand, the surrounding matrix showed a spectrum rather typical of amalgam except for occasional traces of $\mathrm{P}, \mathrm{S}$, and $\mathrm{Cl}$. Figure 12 shows the secondary electron image and $\mathrm{X}$ ray images for $\mathrm{Sn}, \mathrm{Hg}, \mathrm{Ag}$, and $\mathrm{Cu}$ from a large blue-gray crystal. Note the predominance of Sn within the particle, the distribution of $\mathrm{Ag}$ and $\mathrm{Hg}$ in the surrounding matrix, and the traces of $\mathrm{Cu}$ scattered throughout the area.

The microprobe analysis of the white phase observed microscopically confirmed its identity as the $\gamma_{2}\left(\mathrm{Sn}_{8} \mathrm{Hg}\right)$ phase.

Analyses of the amorphous material observed within microscopic cracks of some specimens produced high counts for the $\mathrm{Sn}$ and $\mathrm{Cl}$ X-ray lines. Relatively low counts for $\mathrm{Ag}$ and $\mathrm{Hg}$ were recorded from these areas.

\section{Discussion}

Previous studies of the corrosion of dental amalgam have dealt primarily with the electrochemical behavior of amalgam and its constituent phases in controlled environments. Three reports, ${ }^{5-7}$ however, were identified in the dental literature that dealt with the qualitative effects of clinical corrosion on amalgam restorations. In these studies of extracted teeth, clinical histories were not known. It should be indicated again that the same condition applies to the aged restorations examined in this investigation. However, it is believed that the results of this investigation provide a basis for future studies of the clinical effects of corrosion in which manipulative variables and conditions of placement of the amalgam can be controlled.

The SEM appearance of the amalgam surface and enamel-amalgam interface can be attributed, in part, to the corrosion process. The pitting of the amalgam surface could be the result of leaching out of the corroded $\gamma_{2}$ phase. Porosities can be attributed to incomplete wetting of amalgam powder with $\mathrm{Hg}$ during trituration and to inadequate condensation and finishing of the restorations.

The association of microscopic cracks with porosities is in agreement with the pattern of crack propagation described by Asgar and Sutfin. ${ }^{10}$ In some areas, the cracks were associated with unsupported amalgam along the cavity walls. Portions of the unsupported amalgam seemed to be the result of washed out interfacial corrosion products rather than a deflection of the amalgam margin away from the cavity wall as suggested by Jorgensen. 5 Both mechanisms could be responsible for the marginal ditch in areas of high stress, and either would predispose the amalgam to fracture.

The build up of a corrosion mass at the tooth-amalgam interface and in macroscopic fractures, as shown by the SEM, suggests a dissolution of the amalgam surface. Results of the EM analyses of corrosion products in these areas provide further evidence to this effect. Furthermore, these results support previous observations of $\mathrm{Sn}$ migration from the amalgam to the tooth-amalgam inter- 


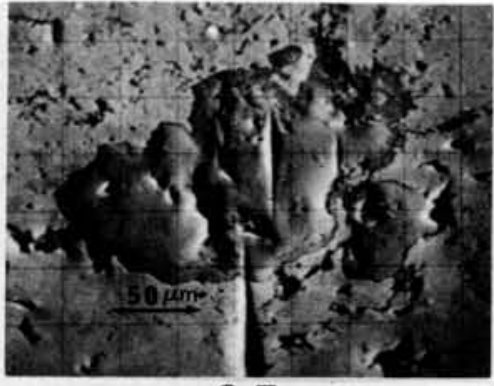

S.E.

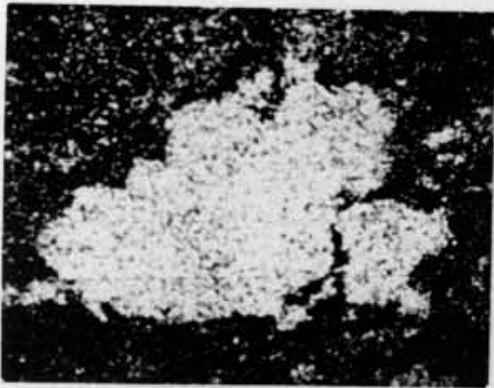

Sn

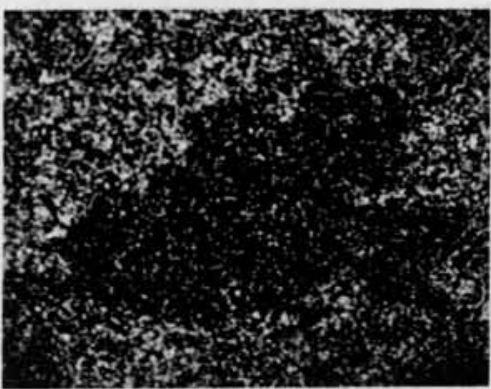

$\mathrm{Ag}$

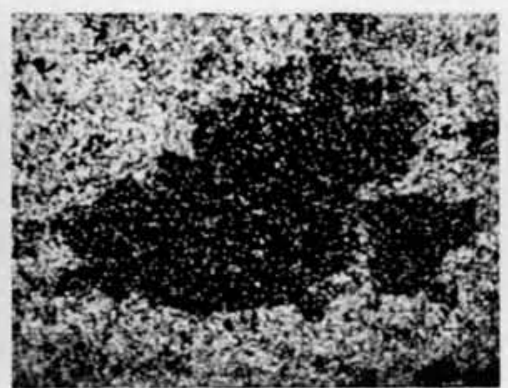

$\mathrm{Hg}$

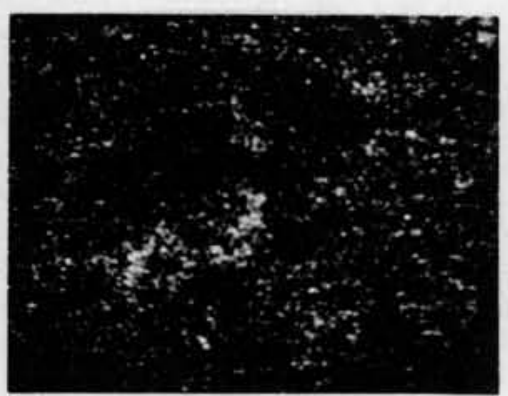

$\mathrm{Cu}$

FIG 12.-Images from electron microprobe analysis of a blue-gray phase (orig mag $\times 500)$.

face $^{7}$ and to the surface of the restoration ${ }^{6}$ in keeping with the theory of selective attack of the $\gamma_{2}$ phase advanced by Jorgensen. ${ }^{5}$ The presence of $\mathrm{Cl}, \mathrm{S}$, and $\mathrm{P}$ in the interfacial corrosion layer reported in this study seems in disagreement with the X-ray diffraction results reported by Mateer and Reitz. ${ }^{6}$ Their study involved analyses of scrapings from external interproximal surfaces and not from the interfacial corrosion layer. It is surprising that the X-ray diffraction analyses contained no patterns for a Sn salt containing $\mathrm{Cl}$.
The altered microstructure throughout the unetched, polished cross-sections indicates the penetrating nature of the corrosion process along voids and the $\gamma_{2}$ phase as proposed by Jorgensen and Saito.11 The decreased quantity and oftentimes absence of $\gamma_{2}$ crystals in areas of severe corrosion further substantiates Jorgensen's ${ }^{5}$ theory that the $\gamma_{2}$ phase is attacked selectively by the corrosion process. The high concentration of $\mathrm{Sn}$ associated with $\mathrm{Cl}$ in the dark-gray areas likewise supports the theory.

The microscopic appearance of the blue- 
gray phase resembled that described by Johnson, Asgar, and Peyton. ${ }^{12}$ However, the phase was more abundant, thus more clearly identifiable, in the aged specimens examined in this study. The clustering of the blue-gray phase along the occlusal surface and the tooth-amalgam interface confirms that the phase in an aged specimen is associated with the corrosion process. The high concentration of $\mathrm{Sn}$ in the blue-gray phase and the distribution of the phase within the amalgam bulk suggests further the relative instability of the $\gamma_{2}$ phase. The Cu concentration of the blue-gray phase was not determined quantitatively. Johnson, Asgar, and Peyton 12 have shown that the structure of the Cu-Sn system in amalgam is $\mathrm{Cu}_{6}-\mathrm{Sn}_{5}$.

It is possible that other nonmetals such as oxygen were present in the corroded phases but were not detected. With the analytical system used in this study, light elements such as those that exist as gases in their pure form, emit X-rays of such low photon energy that the X-rays do not reach the detector of the microprobe. ${ }^{9,13}$

\section{Conclusions}

Within limits of the experimental conditions presented, these conclusions can be made from this investigation: The corrosion process is not confined to the superficial structures of dental amalgam, but rather penetrates throughout the amalgam mass. The $\gamma_{2}$ phase is attacked selectively by the corrosion process yielding $\mathrm{Sn}$ ions that can react with nonmetallic ions present in saliva. Sn released from the $\gamma_{2}$ phase by the corrosion process can migrate to areas within the amalgam that are continuous with the oral environment, such as microscopic cracks, the amalgam surfaces, and the tooth-amalgam interface. In these areas higher chemical activity can occur. The corrosion process yields at least two distinct new phases within the amalgam structure - a dark-gray phase consisting primarily of $\mathrm{Sn}$ and $\mathrm{Cl}$ and a bluegray phase consisting primarily of Sn. The corrosion process yields a corrosion layer at the tooth-amalgam interface consisting of Sn, S, P, and Cl. A controlled study of the clinical effects of corrosion on the constituent phases of dental amalgam is encouraged.

The authors thank L. Allard and J. Rosen for their assistance in the SEM and EM analyses respectively.

\section{References}

1. Guthrow, C.E.; Johnson, L.B.; and LawLEsS, K.R.: Corrosion of Dental Amalgam and Its Component Parts, J Dent Res 46: 1372-1381, 1967.

2. Habu, Hiroyoshi: A Polarographic Study of the Corrosion Tendency of Dental Amalgam, J Nihon Univ Sch Dent 6: 6-16, 1964.

3. Nagai, Kazuo, and OHashi, Masoyoshi: Study of the Corrosion Resistancy of Spherical Amalgam with Reference to Human Saliva, J Nihon Univ Sch Dent 9: 143-163, 1967.

4. Johnson, L.B., and Lawless, K.R.: Corrosion Under Stress of Materials Composing Dental Amalgam, J Biomed Mater Res 3: 569-576, 1969.

5. JoRGENSEN, K.D.: The Mechanism of Marginal Fracture of Amalgam Fillings, Acta Odontol Scand 23: 347-389, 1965.

6. Mateer, R.S., and Reitz, C.D.: Corrosion of Amalgam Restorations, J Dent Res 49: 399-407, 1970.

7. WeI, S.H.Y., and InGram, M.J.: Analyses of the Amalgam-Tooth Interface Using the Electron Microprobe, $J$ Dent Res 48: 317-320, 1969.

8. Allan, F.C.; Asgar, Kamal; and Peyton, F.A.: Microstructure of Dental Amalgam, $J$ Dent Res 44:1002-1012, 1965.

9. Allan, Lawrence, and Rosen, J.J.: Personal communication, June, 1972.

10. Asgar, Kamal, and Sutfin, Lloyd: Brittle Fracture of Dental Amalgam, J Dent Res 44: 977-988, 1965

11. Jorgensen, K.D., and Saito, Tsuyoshi: Structure and Corrosion of Dental Amalgams, Acta Odontol Scand 28: 129-142, 1970.

12. Johnson, L.N.; Asgar, Kamal; and Peyton, F.A.: Microanalysis of Copper-Tin Phases in Dental Amalgam, J Dent Res 48: 872-878, 1969.

13. Modern X-Ray Analysis. Prairie View, Ill: Nuclear Diodes, Inc., 1970. 